\title{
Sistema de economia de fichas associado ao custo da resposta aplicados aos comportamentos de sala de aula de adolescentes com transtorno de déficit de atenção e hiperatividade (TDAH)
}

\author{
Agostinho Jorge Assunção Marafão* \\ Eduardo Chaves Cruz** \\ Rosangela Bertelli***
}

Resumo

O objetivo geral do presente estudo foi avaliar o impacto de um programa de economia de fichas, ou seja, reforçamento positivo, associado à punição negativa, isto é, custo da resposta, sobre o comportamento "fora da tarefa" de dois adolescentes diagnosticados com Transtorno de Déficit de Atenção e Hiperatividade (TDAH). O programa consistia na atribuição ou retirada de pontos consoante a emissão de comportamento adequado ou inadequado à tarefa em execução. O sistema de pontos era efetuado através de cartas individuais, coloridas, numeradas de um a 10 pontos. Também era retirado um ponto, quando o aluno aumentava a frequência de emissão de comportamentos inadequados comparativamente à sessão anterior. Ao atingir 10 pontos, as cartas eram trocadas por privilégios (reforçadores positivos), previamente negociados com o professor. Para testar a igualdade das variâncias utilizou-se a prova de Levene e, para testar a igualdade de médias, utilizou-se o teste $t$ de Student. A significância das diferenças entre as médias foi significativa $(p<0.0001)$ revelando, assim, a eficácia da intervenção sobre o comportamento dos dois participantes. Concluiu-se que a ação convergente dos princípios de reforçamento positivo e punição negativa (custo da resposta) leva à resolução de comportamentos disfuncionais de crianças com TDAH em contexto da sala de aula.

Palavras-chave: Economia de fichas; Reforçamento positivo; Custo da resposta.

http://dx.doi.org/10.5902/1984686X6882

* Escola do Ciclo Preparatório Diogo Cão, Vila Real - Portugal
** Universidade de Trás-os-Montes e Alto Douro, Vila Real - Portugal.
*** Universidade de Trás-os-Montes e Alto Douro, Vila Real - Portugal.

Revista Educação Especial | v. 26 | n. 46 | p. 229-244 | maio/ago. 2013

Santa Maria

Disponivel em: <http://www.ufsm.br/revistaeducacaoespecial> 


\title{
A system of token economy associated to response cost applied to the out of the task behaviour of two adolescents suffering from Attention Deficit Hyperactivity Disorder (ADHD)
}

\begin{abstract}
The aim of this study was to evaluate the impact of a program of token economy, that is, positive reinforcement, associated to negative punishment, that is, response cost, on the "out of the task" behaviour of two adolescents diagnosed as suffering from Attention Deficit Hyperactivity Disorder (ADHD). The program involved presenting or removing points contingent to the emission of adequate or inadequate behaviour, respectively, considering the task at hand. The token economy system was carried out through the adoption of colourful individual cards numbered from one to 10 points. Points were also removed when the student showed at the present session an increased frequence of inadequate behaviour compared to the last session. When reaching 10 points, the cards were exchanged for the privilegies (positive reinforcers) previously negotiated between teacher and student. Statistical treatment was carried out through the use of Levene and Student $t$ tests. The significance of the difference between means $(p<0.0001)$ revealed the efficacy of the intervention treatment on the behaviour of both participants. It was concluded that the merging effect of the positive reinforcement and negative punishment (= response cost) learning principles leads to the modification of classroom disruptive behaviours in children with ADHD.
\end{abstract}

Keywords: Token economy; Positive reinforcement; Response cost.

Introdução

O Transtorno de Déficit de Atenção e Hiperatividade (TDAH) é uma perturbação crônica da infância, caracterizada por um nível de atividade inadequado, baixa tolerância à frustração, impulsividade, fraca organização do comportamento, distração e incapacidade de manter a atenção e a concentração (AMERICAN PSYCHIATRIC ASSOCIATION, 2000). Diferentes tipos de intervenções, com base em modelos comportamentais da aprendizagem, têm sido implementadas com crianças com TDAH (CRUZ; BERTELLI; BIANCHI, 2010; HINSHAW; ERHARDT, 1990; RAPPORT, 1987; WERRY; WOLLERSHEIM, 1989). As intervenções comportamentais no contexto da sala de aula envolvem manipulações dos eventos antecedentes 
ao comportamento-alvo e/ou dos eventos consequentes ao comportamentoalvo, aumentando ou diminuindo a sua frequência da ocorrência.

Em virtude das crianças com TDAH manifestarem comportamentos inadequados, em termos da sua frequência e intensidade, muitas vezes as intervenções comportamentais comprometem-se com o objetivo de aumentar os comportamentos desejáveis, ao mesmo tempo que diminuem os comportamentos indesejáveis (HINSHAW; ERHARDT, 1990). As contingências de reforço às quais as crianças são submetidas permitem selecionar os comportamentos mais apropriados a determinadas situações e, quando isso ocorre, diante de uma dada situação há a expectativa de uma determinada consequência. As consequências que se seguem a determinados comportamentos ativam os neurônios de dopamina, o que equivale a dizer que o cérebro também vive as diferentes contigências de reforço. A ativação da dopamina é essencial para a aprendizagem operante. Aprender a discriminar entre si as situações apropriadas ou não à emissão de determinados comportamentos permite antecipar as diferentes consequências associadas aos diferentes operantes (BERTELLI et al., 2010).

A habilidade de discriminação de estímulos ativa os neurônios de dopamina, ou seja, fornece uma consequência imediata ao nível neural. A criança com TDAH, no entanto, sofre uma deficiência na transferência da ativação dos neurônios de dopamina para a situação que sinaliza as consequências que estariam associadas a determinados comportamentos, isto é, a ativação neural de dopamina é atrasada, retardando assim as consequências do comportamento no nível neural, só ocorrendo, então, após a efetiva consequência contingente ao comportamento. Além dessa ativação tardia da dopamina, a criança com TDAH também sofre de deficiências no escoamento da dopamina ativada, provocando uma presença contínua de dopamina, mesmo após a consequência do seu comportamento, daí o termo Hiperatividade (BERTELLI et al., 2010).

O sistema de gestão de contingências, quando é implementado num ambiente controlado, como a sala de aula, normalmente envolve a utilização do princípio de reforçamento positivo, através da apresentação de pontos contingente ao comportamento desejado. Alguns programas podem também incluir a contingência do custo da resposta (punição negativa), pelo que a criança pode perder pontos por incumprimento das regras ou por emitirem comportamentos indesejáveis. O reforçamento positivo, por um lado, e o custo da resposta, por outro lado, permitem aumentar a frequência de uma resposta desejada, diminuindo a frequência de uma resposta indesejada. O princípio do reforçamento positivo diz respeito à apresentação de uma consequência contingente a um comportamento, gerando um aumento na frequência desse comportamento. A consequência que é apresentada 
contingente ao comportamento é chamada estímulo reforçador. O princípio da punição negativa ou custo da resposta diz respeito à remoção de uma consequência contingente ao comportamento, gerando uma diminuição na frequência desse comportamento. A consequência que é removida contingente ao comportamento é chamada estímulo reforçador. Em outras palavras, ao envolver-se em comportamentos apropriados a criança ganha certos privilégios, porém, ao envolver-se em comportamentos inapropriados, a criança perde certos privilégios (CRUZ; BERTELLI; BIANCHI, 2010).

Normalmente, os comportamentos que são selecionados para serem submetidos ao reforçamento positivo incluem, por exemplo, a criança permanecer centrada na tarefa em execução e concluir o trabalho escolar de forma correta. A contingência de custo da resposta ocorre para inibir o comportamento indesejado ou desviado dos objetivos da tarefa em execução. Os programas de gestão de contingências através de reforçamento positivo e custo da resposta parecem ser bem-sucedidos no aumento da produtividade escolar e do tempo de permanência da criança na tarefa. Um dos programas de modificação de comportamento que tem merecido a atenção dos pesquisadores no âmbito da análise e modificação do comportamento em sala de aula é o programa de economia de fichas associado ao custo da resposta. (BARKLEY, 1989; HINSHAW; ERHARDT, 1990; RAPPORT, 1987; WERRY; WOLLERSHEIM, 1989).

Um programa de modificação de comportamento onde a criança pode ganhar fichas contingente a certos comportamentos e pode trocar essas fichas por consequências reforçadoras back-up é chamado um Sistema de Fichas. Qualquer "coisa" que possa ser acumulada pode ser utilizada como meio de troca em um Sistema de Fichas. A utilização de fichas como estímulos reforçadores condicionados é bastante eficiente, porque fichas podem ser oferecidas imediatamente após a ocorrência do comportamento desejado, podem ser trocadas posteriormente por estímulos reforçadores back-up, e podem, ainda, ser retiradas imediatamente após a ocorrência do comportamento indesejado, o que permite a criação de uma ponte que reduz a distância entre a emissão do comportamento e a consequência apropriada a cada comportamento emitido (BERTELLI; CRUZ; MARAFÃO, 2012).

Os diversos estudos realizados na vertente da gestão comportamental para populações com hiperatividade têm demonstrado que as estratégias que se baseiam exclusivamente nos processos de reforçamento positivo são menos eficazes, se comparadas com a associação do custo da resposta e a consequente aplicação de consequências médias de custo da resposta perante comportamentos inadequados. Para além de reprimendas e reorientações verbais, as penalizações que envolvem a perda ou a retirada de privilégios, pontos ou prêmios contingentes ao comportamento desatento 
e disfuncional mostraram-se úteis quando combinados com procedimentos de reforçamento. O resultado da ação convergente na aplicação desses procedimentos traz diversas vantagens na adoção destas estratégias, que se traduz no aumento dos níveis de comportamento na tarefa, na produtividade do trabalho escolar e na precisão escolar das crianças com hiperatividade, de acordo com as perspectivas de Dupaul, Guevremont e Barkley (1992) e Rapport, Murphy e Bailey (1980; 1982).

O presente estudo procurou analisar o impacto de um programa de economia de fichas, associado com o custo da resposta, sobre um comportamento-alvo (comportamentos fora da tarefa) de dois adolescentes com idades de 14 e 15 anos, que evidenciavam comportamentos disfuncionais e causadores de instabilidade na sala de aula. Para fins de operacionalização de comportamento fora da tarefa, esse foi definido como sendo uma dificuldade em manter a atenção na tarefa $A 1 b$, segundo critério patenteado no "The American Psychiatric Association's Diagnostic and Statistical Manual of Mental Disorders - DSM- IV" (AMERICAN PSYCHIATRIC ASSOCIATION, 2000). Constata-se tal categoria quando o aluno interrompe a atenção na tarefa para envolver-se em outro comportamento. Define-se a atenção como olhar visualmente para os materiais da tarefa. Se o aluno perder o contato visual com os exercícios a executar, então registra-se "comportamento fora da tarefa".

O problema de pesquisa foi formulado da seguinte maneira: Os procedimentos de ensino comportamentais, baseados em programas de economia de fichas associados ao custo da resposta, melhoram o comportamento fora da tarefa da criança hiperativa?

\section{Método}

\section{Participantes}

Os dois participantes, aqui designados João e Carlos, com idade de 14 e 15 anos, apresentavam comportamentos característicos de jovens sofrendo de TDAH.

\section{Características do participante 1}

O participante 1 , aqui designado de João, frequentava o $8^{\circ}$ ano de escolaridade numa escola pública da cidade de Vila Real em Portugal. Encontrava-se ao abrigo do Decreto-lei $n^{\circ} 3 / 2008$ de 7 de Janeiro 
(PORTUGAL, 2008), submetido a um Programa Educativo Individual, onde constavam as medidas inerentes a um Currículo Específico Individual (artigo $21^{\circ}$ ) com Apoio Pedagógico Personalizado (artigo $17^{\circ}$ ) e Adequações no Processo de Avaliação (artigo $20^{\circ}$ ) (PORTUGAL, 2008).

A equipe multidisciplinar de apoio aos alunos com necessidades educativas especiais (NEE) avaliou o jovem em questão, tendo em conta relatórios médicos e técnicos disponíveis no seu processo e destinados ao levantamento de informações referentes às funções do corpo, e efetuou, em consonância com o Conselho de Turma, a avaliação pedagógica destinada à implementação dos aspectos inerentes à atividade e participação, consultando os pais, para estimar a vertente dos fatores ambientais, elementos correspondentes à Classificação Internacional de Funcionalidade (CIF), que servem de métrica à Educação Especial (ORGANIZAÇÃO MUNDIAL DE SAÚDE, 2004).

Atendendo aos registros existentes no seu processo individual, o João apresentava, de acordo com a CIF, uma deficiência nas funções intelectuais (b117.2), nas funções da linguagem oral (b16700.3) e escrita (b16701.3), nas funções de controle dos impulsos, impaciência, tiques e agitação (b1304.3), nas funções da atenção e distração (b140.3), nas funções do temperamento, da personalidade e da amabilidade (b126.3), instabilidade psíquica (b1263.3) e instabilidade emocional (b152.3). Por sua vez, no que diz respeito às Funções Psicomotoras (b147.1), denotavamse dificuldades ligeiras. Evidenciava dificuldades de grau moderado nas Funções Psicossociais Globais (b122.2), Funções da Memória (b144.2) e Funções Cognitivas de Nível Superior (b164.2).

De acordo com os relatórios médicos, também apresentava TDAH (b140.3), encontrando-se associado uma Perturbação de Oposição e Desafio (POD). Evidenciava instabilidade, problemas de conduta, agressividade, auto e heteroagressão verbal, baixa tolerância à frustração (b126.3). Tinha dificuldade em iniciar e terminar uma tarefa, a realização de uma tarefa simples era prolongada no tempo e, perante duas situações de opção, o João revelava hesitação na tomada de decisão (d177.2). Na situação de realização de tarefas múltiplas (d220.2), manifestava lacunas na realização de uma só tarefa sem a ajuda de alguém, caso essa tarefa fosse escolar. Revelava dificuldades em compreender e seguir regras e orientar-se no relacionamento interpessoal e na convivência.

Às dificuldades de comportamento assinaladas estavam associados problemas de aprendizagem nas áreas educacionais básicas, com predominância para o cálculo (d172.3) e escrita (d170.3). Apresentava ainda dificuldade em focar a atenção nas tarefas (d160.3). 
Relativamente aos fatores ambientais, o João tinha o apoio dos professores do Conselho de Turma, do docente de Educação Especial, do Diretor de Turma e dos Serviços de Psicologia, tendo em conta a orientação, o apoio e a organização do processo do João (e360+4), (e575+3). O João ainda beneficiava do apoio social geral e relacionado com prestadores de cuidados pessoais (e340+3). A família próxima não funcionava como facilitadora, devido à ausência dos pais, à situação familiar (e310.3) e às atitudes individuais dos membros da família próxima (e410.3).

\section{Características do participante 2}

O participante 2 , aqui designado de Carlos, frequentava o $8^{\circ}$ ano de escolaridade numa escola pública da cidade de Vila Real, Portugal, inserido num Programa Educativo Individual, ao abrigo do Decreto-Lei $n^{\circ} 3 / 2008$, de 7 de Janeiro (PORTUGAL, 2008). De acordo com as suas características, foram-Ihe aplicadas as medidas de regime educativo Apoio Pedagógico Personalizado (artigo $17^{\circ}$ ), Adequações Curriculares Individuais (artigo 18 ${ }^{\circ}$ ) e Adequações no Processo de Avaliação (artigo $20^{\circ}$ ).

Conforme a classificação que suporta a legislação acima mencionada e determina a inclusão ou não dos alunos na Educação Especial, a equipe multidisciplinar considerou os relatórios existentes, todos os elementos inerentes às funções do corpo, atividade e participação e ainda os fatores ambientais que a CIF determina para descrever em relatório Técnicopedagógico as características que se enunciam relativamente ao participante 2. O relatório médico assinalava problemas de TDAH (b140.3), problemas da esfera emocional (b152.3), perturbações específicas de aprendizagem e problemas de coordenação motora (b1478.3). Não apresentava problemas nas funções intelectuais (b117.0).

De forma associada e relativamente às Perturbações Específicas da Aprendizagem, o Carlos foi diagnosticado com um quadro de dislexia de grau acentuado (b1678.3), com comprometimento no desempenho da leitura e da escrita. Apresentava dificuldades moderadas ao nível da memória de trabalho verbal, que interferiam significativamente com a sua capacidade para aprender e executar as tarefas escolares (b1440.3 e b1448.3), dificuldades em repetir sequências numéricas seguindo uma ordem específica, acentuandose nas tarefas de reter e realizar operações cognitivas sobre informações fonológicas (b1140.3 e b1448.3). O Carlos apresentava ainda cansaço e dificuldades na capacidade de reter com precisão os pormenores fonológicos das palavras (b1678.2). 
$\mathrm{Na}$ leitura surgiam lacunas na compreensão de pormenores dos textos, com falhas ao nível da decodificação e da metacognição (b16701.3). $\mathrm{Na}$ escrita, as dificuldades de análise fonêmica eram acentuadas, com erros considerados primitivos, tendo em conta o seu nível de escolaridade, ausência de padrões ortográficos sublexicais específicos da ortografia e interiorização de conhecimentos lexico-ortográficos, mesmo em palavras mais frequentes. Evidenciava dificuldades na monitorização da escrita, palavras incompletas e várias formas ortográficas para a mesma palavra (b16711.3). Para além das dificuldades descritas ao nível das funções do corpo, o Carlos apresentava dificuldades respeitantes à atividade e participação. Neste capítulo da CIF, a equipe multidisciplinar e os relatórios confirmavam as dificuldades do Carlos, que apresentava também dificuldades em se desligar dos ruídos que o distraíam, para concentrar a atenção em estímulos específicos, sendo-lhe muito difícil manter intencionalmente a atenção em tarefas específicas durante um intervalo de tempo razoável (d160.3). Revelava erros na interpretação de enunciados, tanto em questões simples como em questões compostas (b1720.3).

Relativamente aos fatores ambientais, o Carlos estava perfeitamente integrado na turma. Tomava diariamente a medicação destinada à atividade motora e à distração (e1101+2). A mãe era extremamente atenta e valorizava os progressos do filho $(\mathrm{e} 310+3)$. Os professores eram muito empenhados, fazendo as adequações necessárias às aprendizagens do aluno (e360+3).

\section{Instrumentos}

Em função do problema de pesquisa previamente formulado, os instrumentos de coleta de dados selecionados foram os seguintes:

a) critérios específicos de diagnóstico de TDAH do DSM-IV, que foram aplicados para detectar os comportamentos hiperativos em contexto escolar;

b) grelha de observação para registro dos comportamentos hiperativos no contexto escolar em situação de apoio individualizado, no PréTeste e no Pós-Teste, que teve como objetivo determinar os comportamentos hiperativos mais salientes no contexto de sala de aula, de forma a identificar os comportamentos a serem modificados através do programa de reforçamento positivo associado ao custo da resposta; 
c) ficha de diagnóstico de preferências, destinada a identificar as preferências pessoais dos participantes, tendo como objetivo a aplicação dos privilégios, após a obtenção dos 10 pontos estipulados;

d) escala de apoio à observação de comportamentos no pré e póstestes, tipo escala de Likert, com parâmetros definidos em cada sessão, abarcando os itens Nunca (0 observações ou registros), Um Pouco (1 a 9 observações ou registros), Frequentemente (10 a 29 observações ou registros) e Muito Frequente (30 a 40 observações ou registros);

e) cartas coloridas numeradas de um a 10 pontos, de apoio ao programa de reforçamento positivo associado ao custo da resposta.

\section{Procedimento}

A aplicação do programa foi feita ao longo dos meses de janeiro, fevereiro e março, com duas sessões semanais, cada uma com a duração de 20 minutos. O observador, utilizando um cronômetro para observar os comportamentos, realizava apenas um registro, relativamente ao número de vezes em que o comportamento era observado durante cada período de 30 segundos. A questão fundamental na elaboração e aplicação de um programa de economia de fichas associado ao custo da resposta era determinar se os procedimentos do programa reduziam com eficiência os comportamentos hiperativos e favoreciam o incremento escolar no contexto da sala de aula.

O programa baseava-se na análise dos comportamentos dos participantes de acordo com a escala de observação. Os participantes eram observados na sala de apoio durante um período de 20 minutos, quando se encontravam a trabalhar individualmente, na carteira, efetuando as tarefas escolares que o professor estipulava e de acordo com a planificação (leitura, escrita, cálculo e exercícios de promoção cognitiva). Focalizava-se a importância de manter ocupado o educando, com exercícios no tempo de observação. O docente emitia a informação indicando o lugar na carteira e, permanecendo sentado, o aluno executava o trabalho solicitado. Depois de estar sentado, iniciava-se o processo de observação e registro de comportamentos, considerando os procedimentos abaixo.

Inicialmente era efetuada uma explicação aos alunos participantes sobre as regras que se consideravam e dos comportamentos mais importantes que seriam ou não permitidos. Os comportamentos não permitidos tinham que incluir a categoria estabelecida na ficha de observação para registros de comportamentos fora da tarefa. 
O programa de economia de fichas associado ao custo da resposta consistia na atribuição ou retirada de pontos. O sistema de pontos era efetuado através de cartas individuais, coloridas, numeradas de um a 10 pontos e, em cada sessão, quando o aluno apresentava comportamentos adequados (Nunca (0) ou Um Pouco (1)), atribuía-se um ponto (carta) para a categoria de interesse (fora da tarefa). Quando os comportamentos eram inadequados, retirava-se um ponto (carta) para a categoria de interesse, de acordo com a escala de itens acima mencionada. Também era retirado um ponto, quando o aluno aumentava a frequência de emissão de comportamentos inadequados comparativamente à sessão anterior e, quando o aluno atingia 10 pontos, as cartas eram trocadas por privilégios que iam de encontro ao que havia sido estabelecido mediante as preferências do aluno, previamente negociadas com o professor. Estabeleceram-se, assim, critérios para a obtenção rápida das fichas e privilégios associados às mesmas.

O programa de economia de fichas associado ao custo da resposta foi implementado durante o segundo período letivo, com duas sessões semanais de trabalho. Cada sessão abrangia 20 minutos de observação, num bloco de 45 minutos. No final de cada sessão o professor dialogava com o aluno para que se analisassem e se compreendessem os resultados. Procedia-se, então, às explicações relativamente aos progressos e/ou retrocessos comportamentais.

\section{Resultados}

Em função da operacionalização das variáveis analisadas e do problema de pesquisa colocado foi efetuada a comparação das médias dos registros, antes e após o tratamento. Para o efeito, aplicou-se inicialmente o teste de Levene para confrontar a homogeneidade de variâncias, ou seja, para testar a igualdade das variâncias e, então, o teste-T, com o objetivo de comparar as médias Optou-se por uma análise e descrição mais ordenadas e detalhadas, descrevendo em separado os resultados do Participante 1 e os resultados do Participante 2.

No que respeita ao Participante 1 , a Tabela 1 mostra que, na fase de pré-teste, no decurso das seis sessões em que o comportamento Fora da Tarefa foi observado, a média dos registros desse comportamento situou-se em 33.16, com um desvio padrão de 2.78. Após a aplicação do programa, ao longo das 22 sessões, constatou-se um registro da modificação do comportamento Fora da Tarefa, com uma média de 7.18 e um desvio padrão de 3.68. A diferença média de 25.98 encontrada entre o valor de 33.16 e 7.18, evidenciada na Tabela 2, é estatisticamente significativa ( $p<$ 
0.001) e reveladora da eficácia do tratamento. Deste modo, os resultados assinalam que o programa, relativamente ao Participante 1 e no que respeita ao comportamento Fora da Tarefa, produziu um impacto positivo e significativo, tendo em conta o declínio observado no número de registros do comportamento efetuado na fase de pós-teste.

Tabela 1. Pré-teste e pós-teste - participante 1

\begin{tabular}{|c|c|c|c|}
\hline Fases & número de sessões & média aritmética & desvio padrão \\
\hline pré-teste & 6 & 33.16 & 2.78 \\
\hline pós-teste & 22 & 7.18 & 3.68 \\
\hline
\end{tabular}

Tabela 2. Teste com amostras independentes - participante 1

\begin{tabular}{|c|c|c|c|c|c|c|c|}
\hline \multicolumn{2}{|c|}{} & \multicolumn{2}{c|}{$\begin{array}{c}\text { teste de Levene } \\
\text { para a igualdade de } \\
\text { variâncias }\end{array}$} & \multicolumn{4}{|c|}{ teste t para a igualdade de médias } \\
\cline { 2 - 7 } & $\mathrm{F}$ & significância & $\mathrm{t}$ & $\begin{array}{c}\text { graus de } \\
\text { liberdade }\end{array}$ & significância & $\begin{array}{c}\text { diferença } \\
\text { média }\end{array}$ \\
\hline $\begin{array}{c}\text { fora da } \\
\text { tarefa }\end{array}$ & $\begin{array}{c}\text { variâncias } \\
\text { iguais }\end{array}$ & 0.302 & 0.587 & 15.980 & 26 & 0.000 & 25.98 \\
\hline & $\begin{array}{c}\text { variâncias } \\
\text { desiguais }\end{array}$ & - & - & 18.793 & 10.347 & 0.000 & 25.98 \\
\hline
\end{tabular}

Em relação ao Participante 2, submetido ao mesmo número de sessões que o Participante 1 , nas duas fases pode-se observar através da Tabela 3, que a média dos registros do comportamento Fora da Tarefa foi de 13.16, com um desvio padrão de 2.85 , na fase de pré-teste e que na fase de pós-teste com a aplicação do programa, os registros do comportamento desceram para um valor médio de 5.63, com um desvio padrão de 2.49. Mais uma vez, a diferença média de 7.53, estatisticamente significativa $(p<0.001)$, apresentada na Tabela 4 evidencia que o programa foi eficiente. Pode-se afirmar, tal como para o Participante 1, que o programa também revelou-se eficaz para o Participante 2 em termos da modificação do comportamento Fora da Tarefa.

Tabela 3. Pré-teste e pós-teste - participante 2

\begin{tabular}{|c|c|c|c|}
\hline Fases & número de sessões & média aritimética & desvio padrão \\
\hline pré-teste & 6 & 13.16 & 2.85 \\
\hline pós-teste & 22 & 5.63 & 2.49 \\
\hline
\end{tabular}


Agostinho Jorge Assunção Marafão - Eduardo Chaves Cruz - Rosangela Bertelli

Tabela 4. Teste com amostras independentes - participante 2

\begin{tabular}{|c|c|c|c|c|c|c|c|}
\hline \multicolumn{2}{|c|}{} & \multicolumn{2}{|c|}{$\begin{array}{c}\text { teste de Levene } \\
\text { para a igualdade de } \\
\text { variâncias }\end{array}$} & \multicolumn{4}{|c|}{ teste t para a igualdade de médias } \\
\cline { 2 - 7 } & $\mathrm{F}$ & significância & $\mathrm{t}$ & $\begin{array}{c}\text { graus de } \\
\text { liberdade }\end{array}$ & significância & $\begin{array}{c}\text { diferença } \\
\text { média }\end{array}$ \\
\hline $\begin{array}{c}\text { fora } \\
\text { da } \\
\text { tarefa }\end{array}$ & $\begin{array}{c}\text { variâncias } \\
\text { iguais }\end{array}$ & 0.015 & 0.904 & 6.358 & 26 & 0.000 & 7.53 \\
\hline & $\begin{array}{c}\text { variâncias } \\
\text { desiguais }\end{array}$ & - & - & 5.871 & 7.227 & 0.001 & 7.53 \\
\hline
\end{tabular}

Relativamente à análise conjunta dos resultados, a Tabela 5 apresenta as sessões correspondentes aos dois participantes na fase de pré-teste $(6+6=12$ sessões $)$ e as sessões do pós-teste $(22+22=44$ sessões). A coluna das médias traduz, em relação aos dois participantes na fase do pré-teste, o valor de 23.16 registros do comportamento Fora da Tarefa, enquanto na fase do pós-teste, com a aplicação do programa de economia de fichas associado com a convergência do custo da resposta, a categoria do comportamento Fora da Tarefa baixou para uma média de 6.40 registros. Da diferença constatada através da subtração dos 23.16 registros do comportamento Fora da Tarefa no pré-teste e os 6.40 registros do mesmo comportamento no pós-teste, resultou uma diferença média de 16.76 registros. Assim, relativamente à Tabela 6, rejeitou-se a igualdade de variâncias $(p<0.05)$, pelo que a leitura da significância das diferenças entre as médias é lida na segunda linha e que é altamente significativa ( $p$ $<0.0001$ ). Verifica-se pelos dados apresentados que, com o tratamento, os participantes permaneceram mais tempo em contato visual com a tarefa.

Tabela 5. Pré-teste e pós-teste - ambos participantes

\begin{tabular}{|c|c|c|c|}
\hline Fases & número de sessões & média aritimética & desvio padrão \\
\hline pré-teste & 12 & 23.16 & 10.78 \\
\hline pós-teste & 44 & 6.40 & 3.20 \\
\hline
\end{tabular}


Tabela 6. Teste com amostras independentes - ambos participantes

\begin{tabular}{|c|c|c|c|c|c|c|c|}
\hline \multicolumn{2}{|c|}{} & \multicolumn{2}{|c|}{$\begin{array}{c}\text { teste de Levene } \\
\text { para a igualdade de } \\
\text { variâncias }\end{array}$} & \multicolumn{4}{|c|}{ teste t para a igualdade de médias } \\
\cline { 2 - 7 } & $\mathrm{F}$ & significância & $\mathrm{t}$ & $\begin{array}{c}\text { graus de } \\
\text { liberdade }\end{array}$ & significância & $\begin{array}{c}\text { diferença } \\
\text { média }\end{array}$ \\
\hline $\begin{array}{c}\text { fora } \\
\text { da } \\
\text { tarefa }\end{array}$ & $\begin{array}{c}\text { variâncias } \\
\text { iguais }\end{array}$ & 113.93 & 0.00 & 9.11 & 54 & 0.000 & 16.76 \\
\hline & $\begin{array}{c}\text { variâncias } \\
\text { desiguais }\end{array}$ & - & - & 5.31 & 11.53 & 0.000 & 16.76 \\
\hline
\end{tabular}

\section{Discussão e conclusão}

O objetivo geral do estudo foi avaliar o impacto do programa de economia de fichas associado ao custo da resposta sobre o comportamento Fora da Tarefa de dois adolescentes diagnosticados com TDAH. Os resultados obtidos, através das análises estatísticas efetuadas, demonstraram que o participante 1, na fase de pré-teste, apresentava 33.16 registros e, na fase de pós-teste, os resultados foram de 7.18. A diferença média entre as duas fases foi de 25.98, o que refletiu uma clara diminuição de registros e a consequente eficácia do programa. Para o participante 2 foram aplicadas as mesmas sessões e, relativamente à fase de pré-teste, o resultado dos registros obtidos situavam-se em 13.16 e, na fase de pós-teste, o resultado foi de 5.63 registros. A constatar pela diferença média de 7.53 , mais uma vez, confirmou-se a eficácia da intervenção. No somatório de todas as sessões, para ambos os participantes, e de modo simétrico, os resultados no préteste situaram-se em 23.16 registros e, na fase de pós-teste, os registros passaram para 6.40. A eficácia relativa à intervenção comportamental foi comprovada através da diferença média de 16.76 comparativamente às duas fases. Estatisticamente, portanto, confirmou-se a eficácia da aplicação do programa.

Os resultados obtidos dão suporte a outros estudos assinalados na literatura, que, de forma combinada ou isolada, os programas de modificação de comportamento, através de economia de fichas associada ao custo da resposta, produziram efeitos positivos nas variáveis dependentes estudadas. Por exemplo, os estudos de caso individual que utilizaram o custo da resposta em contextos escolares produziram efeitos significativos na modificação do comportamento (ATKINS; PELHAM; WHITE, 1989; KELLEY; MCCAIN, 1995; RAPPORT; MURPHY; BAILEY, 1982). Rapport, Murphy e Bailey (1982) assinalaram melhorias no comportamento na tarefa e em permanecer 
sentado no lugar com percentagens de $30 \%$ a $50 \%$ durante as linhas de base para percentagens de $80 \%$ a $100 \%$ com a introdução do programa de custo da resposta. Na mesma direção, a investigação demonstrou a eficácia do reforçamento através de economia de fichas com alunos apresentando alta e baixa incidência de dificuldades (CHRISTENSEN; YOUNG; MARCHANT, 2004; KAZDIN; GEESEY, 1977). Por seu lado, Simonsen et al. (2008) identificaram o programa de reforçamento com fichas como uma das 20 práticas de gestão da sala de aula empiricamente validadas.

O reforçamento positivo e o custo da resposta (punição negativa) são importantes métodos de modificação comportamental em crianças com TDAH. Para uma maior compreensão da investigação dos efeitos da contingência de reforçamento positivo na frequência de ocorrência e intensidade dos comportamentos, nomeadamente na forma como podem ser geridos, Luman, Oosterlaan e Sergeant (2005) analisaram 22 estudos correspondentes a 1181 crianças com TDAH, em que foram aplicadas técnicas de contingência de reforço, evidenciando a sua eficácia ao nível da realização das tarefas, da motivação e no plano psicofisiológico. Os resultados do estudo indicaram que as contingências de reforço tiveram um impacto significativo na realização das tarefas e nos níveis motivacionais das crianças com TDAH.

Avalidade empírica dos programas de economia de fichas associados ao custo da resposta, tanto no presente estudo quanto em estudos anteriores assinalados na literatura, evidenciam que a aplicação destas técnicas operantes resultam em ganhos significativos para os participantes. Concluiuse, portanto, que os professores do ensino regular e do ensino especial, que no dia-a-dia trabalham com crianças com TDAH, podem encontrar nesses programas uma ferramenta eficaz para a gestão dos comportamentos de sala de aula e um meio de melhorar os desempenhos cognitivos das crianças, através da reeducação dos seus níveis atencionais e motivacionais.

\section{Referências}

AMERICAN PSYCHIATRIC ASSOCIATION. Diagnostic and Statistical Manual of Mental Disorders. 4th ed. Washington, DC: Author, 2000.

ATKINS, M. S.; PELHAM, W. E.; WHITE K. J. Hyperactivity and attention deficit disorders. In: Hersen, M. (Ed.). Psychological aspects of developmental and physical disabilities: a casebook. California: Sage Publications, 1989. p. 137-156. 
BARKLEY, R. A. Attention deficit-hyperactivity disorder. In: Mash, E. J. \& Barkley, R. A. (Eds.). Treatment of childhood disorders. New York: Guilford, 1989. p. 39-72.

BERTELLI, R.; CRUZ, E. C.; VASCONCELOS-RAPOSO, J.; FERNANDES, H. M.; BIANCHI, J. J. P. Neurobiologia da Hiperatividade. In: JORNADAS A HIPERATIVIDADE E OS DÉFICES DE ATENÇÃO E CONCENTRAÇÃO. 2010. Vila Real, Portugal: Centro de Investigação em Desporto, Saúde e Desenvolvimento Humano e Centro Psicológico e Terapêutico "Encantado": p. sem número. $1 \mathrm{CD}-\mathrm{ROM}$.

BERTELLI, R.; CRUZ, E. B. C.; MARAFÃO, A. Economia de fichas. In: JORNADAS PEDAGÓGICAS: O PROFESSOR FAZ A DIFERENÇA NO DESEMPENHO ESCOLAR. 2012, Vila Real, Portugal: Universidade de Trásos-Montes e Alto Douro: p. sem número. 1 CD-ROM.

CHRISTENSEN, L.; YOUNG, K. R.; MARCHANT, M. The effects of a peermediated positive behavior support program on socially appropriate classroom behavior. Education and Treatment of Children, v. 27, p. 199-234, 2004.

CRUZ, E. C.; BERTELLI, R.; BIANCHI, J. J. P. Perspectivas recentes no tratamento do Transtorno de Déficit de Atenção e Hiperatividade. Revista de Educação Especial, v. 23, n. 36, p. 11-26, 2010.

DIREÇÃO-GERAL DE INOVAÇÃO E DE DESENVOLVIMENTO CURRICULAR. Manual de Apoio à Prática. Ministério da Educação. Lisboa: Autor, 2008.

DUPAUL, G. J.; GUEVREMONT, D. C.; BARKLEY, R. A. Behavioral treatment of attention-deficit hyperactivity disorder in the classroom: The use of the Attention Training System. Behavior Modification, v. 16, p. 204-225, 1992.

HINSHAW, S. P.; ERHARDT, D. Behavioral treatment of attention deficithyperactivity disorder. In: Van Hasselt, V. B. \& Hersen, M. (Eds.). Handbook of behavior therapy and pharmacotherapy for children: an integrative approach. New York: Plenum, 1990.

KAZDIN, A. E.; GEESEY, S. Simultaneous-treatment design comparisons of the effects of earning reinforcers for one's peers versus oneself. Behavior Therapy, v. 8, p. 682-693, 1977. 
KELLY, M. L.; MCCAIN, A. P. Promoting academic performance in inattentive children: the relative efficacy of school-home notes with and without response cost. Behavior Modification, v. 19, p. 76-85, 1995.

LUMAN, M.; OOSTERLAAN, J.; SERGEANT, J. A. The impact of reinforcement contingencies on AD/HD: a review and theoretical appraisal. Clinical Psychology Review, v. 25, p. 183-213, 2005.

ORGANIZAÇÃO MUNDIAL DA SAÚDE. Classificação internacional de funcionalidade, incapacidade e saúde. Lisboa: Direção-Geral da Saúde, 2004.

PORTUGAL. Decreto-lei n. ${ }^{\circ}$ 3/2008, Diário da República, $1^{\text {a }}$ Série - N. ${ }^{\circ} 4,7$ de 7 de janeiro 2008.

RAPPORT, M. D. Attention deficit disorder with hyperactivity. In: Hersen, M. \& Van Hasselt, V. B. (Eds.). Behavior therapy with children and adolescents: A clinical approach. New York: Wiley, 1987, p. 325-361

RAPPORT, M. D.; MURPHY, H. A.; BAILEY, J. S. The effects of a response cost treatment tactic on hyperactive children. Journal of School Psychology, v. 18, p. $98-111,1980$.

RAPPORT, M. D.; MURPHY, H. A.; BAILEY, J. S. Ritalin versus response cost in the control of hyperactive children: A within-subject comparison. Journal of Applied Behavior Analysis, v. 15, p. 205-216, 1982.

SIMONSEN, B.; FAIRBANKS, S.; BRIESCH, A.; MYERS, D.; SUGAI, G. Evidenced-based practices in classroom management: Considerations for research to practice. Education and Treatment of Children, v. 31, p. 351380, 2008.

WERRY, J. S.; WOLLERSHEIM, J. P. Behavior therapy with children and adolescents: A 20-year overview. Journal of the American Academy of Child and Adolescent Psychiatry, v. 28, p. 1-18, 1989.

\section{Correspondência}

Eduardo Chaves Cruz - Universidade de Trás-os-Montes e Alto Douro, Escola de Ciências Humanas e Sociais, Departamento de Educação e Psicologia, Edifício do CIFOP.

Rua Dr Manuel Cardona, Apartado 1013, Vila Real - 5001-558, Vila Real - Portugal.

E-mail: echaves@utad.pt

Recebido em 19 de outubro de 2012

Aprovado em 01 de dezembro de 2012 